\title{
Nitric Oxide Modulates Local Reflexes of the Tailfan of the Crayfish
}

\author{
Makoto Araki, ${ }^{*}$ Hansjürgen Schuppe, Sawako Fujimoto,, Toshiki Nagayama,* \\ Philip L. Newland \\ School of Biological Sciences, University of Southampton, Biomedical Sciences Building, Bassett \\ Crescent East, Southampton SO16 7PX, United Kingdom
}

Received 17 September 2003; accepted 5 November 2003

\begin{abstract}
Electrical stimulation of sensory neurons that innervate receptors on the tailfan of crayfish evokes a reflex response of motor neurons that produce movements of the blades of the tailfan, the uropods. We analyzed the modulatory effects of nitric oxide (NO) on the spike frequency of the reflex response. Bath application of $L$-arginine and SNAP, which elevate endogenous and exogenous NO levels, increased the frequency of the evoked response, whereas the application of $\mathrm{L}$ NAME and PTIO, which reduce NO levels, decreased the frequency of the response. To determine through what pathway and target NO exerted these effects we bath applied ODQ, an inhibitor of soluble guanylyl cy-
\end{abstract}

\section{INTRODUCTION}

The unorthodox messenger, nitric oxide (NO), serves a key role in the normal function of the central nervous system. A number of studies have shown that NO is potentially neurotoxic and can induce neuronal death (Schmidt and Walter, 1994), while others have shown that NO may also play a neuroprotective role

*Permanent address: Division of Biological Sciences, Graduate School of Science, Hokkaido University, Sapporo 060, Japan.

Correspondence to: P.L. Newland (pln@soton.ac.uk).

Contract grant sponsor: Yamada Science Foundation (T.N. and P.L.N.).

Contract grant sponsor: Biotechnology and Biological Sciences Research Council (UK) (P.L.N.).

Contract grant sponsor: Ministry of Education, Science, Sport, Culture and Technology (Japan) (T.N.).

(C) 2004 Wiley Periodicals, Inc.

Published online 16 April 2004 in Wiley InterScience (www. interscience.wiley.com).

DOI 10.1002/neu.20007 clase (sGC), which decreased the frequency of response, and 8-br-cGMP, which increased the spike frequency of response. To provide further evidence that $\mathrm{NO}$ acts via sGC, we elevated NO levels with $L$-arginine while simultaneously inhibiting sGC with ODQ. This application reduced the response to control levels, indicating that NO in the terminal ganglion of crayfish acts via SGC to modulate cGMP levels, which in turn regulate the responses of the uropod motor neurons. () 2004 Wiley Periodicals, Inc. J Neurobiol 60: 176-186, 2004

Keywords: cyclic GMP; modulation; motor neuron; reflex; local circuits

(Kiedrowski et al., 1992; Wink et al., 1993). Under normal physiological conditions, however, NO acts as a neurotransmitter, and what makes it unorthodox is that it is a small nonpolar molecule that diffuses from its site of synthesis in three dimensions (Philippides et al., 2000).

$\mathrm{NO}$ is synthesized from L-arginine in a $\mathrm{Ca}^{2+} / \mathrm{cal}-$ modulin-dependent process by the enzyme nitric oxide synthase (NOS), in a reaction requiring oxygen and NADPH (Moncada et al., 1991; Stuehr and Griffith, 1992). NO can then act on one of its molecular targets, soluble guanylyl cyclase (sGC), resulting in the production of cGMP, which in turn may act on cGMP-dependent protein kinase to phosphorylate downstream target proteins to regulate neuron responses (Bredt and Snyder, 1989). NO has been implicated in the modulation of the local circuits and network properties of many integrative systems, including the olfactory system (Breer and Shepherd, 
1993; Gelperin et al., 2001; Kendrick et al., 1997), the visual system (Bacigalupo et al., 1995; Cudeiro and Rivadulla, 1999; Elphick et al., 1996; Schmachtenberg and Bicker, 1999), and the mechanosensory system (Schuppe et al., 2001a; Ott and Burrows, 1998; Ott et al., 2000), as well as playing a key role in memory formation in many animals (see for example Kemenes et al., 2002; Müller, 1996). In the crayfish, $\mathrm{NO}$ is involved in modulating the activity of local circuits that control and generate movements of the appendages of the tailfan, at many different levels. Nicotinamide adenine dinucleotide phosphate diaphorase (NADPHd) histochemistry combined with intracellular double labeling of identified interneurons has revealed the identity of many of the neurons that contain the enzyme NOS and synthesize NO, as intersegmental ascending interneurons with somata in the terminal abdominal ganglion (Schuppe et al., 2001b). Imaging studies with a NO-specific indicator (Schuppe et al., 2002) have directly shown that NO is synthesized endogenously in the terminal abdominal ganglion and that NOS inhibitors can reduce the level of NO synthesis. Conversely, providing additional substrate for its synthesis enhances endogenous NO release (Schuppe et al., 2002). Pharmacological studies have shown that NO modulates presynaptic inhibition of exteroceptive afferents, a mechanism that reduces sensory inflow in local circuits (Schuppe and Newland, 2004). NO also modulates synaptic inputs onto ascending interneurons, by either enhancing or reducing their inputs depending on the identity of the interneuron (Aonuma and Newland, 2001). Furthermore, mechanosensory inputs onto spiking local interneurons in these same local circuits are also modulated by NO (Aonuma and Newland, 2002), in addition to NO-mediated effects on the dynamic properties of the inputs to ascending interneurons (Schuppe et al., 2004).

While we know that NO modulates the outputs of many neural networks, such as those involved in swimming in Xenopus (McLean and Sillar, 2000), feeding in the locust (Rast, 2001), or in producing stomach movements of lobsters (Scholtz et al., 2001), because of the multiplicity of effects of $\mathrm{NO}$ action on mechanosensory processing, the exact role it may have on the outputs of local circuits remains unclear. Moreover, the molecular targets of NO in the terminal ganglion have yet to be clearly identified. Stimulation of sensory receptors on, or in, the tailfan of crayfish leads to local reflex movements of its appendages, the uropods, comprising the exopodite, endopodite, and protopodite (Nagayama, 1997; Newland, 2001) that form part of the normal behavioral repertoire of crayfish. The aim of this study was therefore to analyze the effects of $\mathrm{NO}$ on these local reflexes and to determine through what target $\mathrm{NO}$ exerts its effects in the terminal abdominal ganglion.

\section{MATERIALS AND METHODS}

Experiments were performed on adult crayfish, Pacifastacus leniusculus (Dana), of both sexes and of $8-12 \mathrm{~cm}$ body length (rostrum to telson), at room temperature $\left(20-24^{\circ} \mathrm{C}\right)$. Crayfish were obtained locally from a commercial supplier (Riversdale Farm, Dorset, UK) and maintained in freshwater tanks until use.

For experiments, crayfish were quickly decapitated and the distal part of the abdomen, from the third to the terminal segment, removed and pinned securely in a Sylgard lined chamber, $10 \mathrm{ml}$ in volume, containing physiological saline (van Harreveld, 1936). The terminal abdominal ganglion was then exposed by cutting through the lateral margins of the overlying sixth ventral sternite, and the surrounding soft cuticle, connective tissue and ventral aorta were all removed. The dissection was then extended into the protopodite to reveal the bifurcation points of nerves 2 and 3 into their respective sensory and motor bundles. A silver wire extracellular hook electrode was placed on the nerve 2 sensory bundle for stimulation, and its motor bundle then cut. A second hook electrode was placed on the motor bundle of nerve 3 for recording, and the motor bundle cut distal to the electrode along with its sensory bundle. We could, therefore, stimulate sensory neurons in nerve 2 and record the reflex responses of motor neurons in nerve 3 , which include those that innervate the ventral rotator muscles and the lateral, dorsal, and slow promotor (opener) muscles (Larimer and Kennedy, 1969). Nerve 2 sensory neurons were stimulated 10 times, for $1 \mathrm{~ms}$ duration, with an interstimulus interval of $100 \mathrm{~ms}$. Groups of stimuli were then repeated at 2 min intervals throughout the course of an experiment. The stimulus intensity was set just above threshold for evoking reflex responses in nerve 3 motor neurons in order to reduce to a minimum any summation of motor spikes during stimulation. In the few cases where summation did occur individual spikes could still be discriminated in the traces so that spike number could still be counted. Spike frequency was calculated for the $100 \mathrm{~ms}$ period following electrical stimulation.

All recordings were displayed on a Tektronix digital oscilloscope and stored on computer using Cambridge Electronic Design (CED; UK) 1401 analogue-to-digital interface using Spike 2 software (CED; UK). Data were analyzed off-line, again using Spike 2, and were based on recordings from 56 crayfish. Statistical significance was analyzed using two-way analysis of variance (ANOVA) and the Pearson chi-square test.

\section{Bath Application of Drugs}

All pharmacological agents were obtained from Sigma Chemical Co. Ltd. and included D-arginine, L-arginine, $\mathrm{N}^{\mathrm{G}}$ - 
nitro-D-arginine methyl ester (D-NAME), $\mathrm{N}^{\mathrm{G}}$-nitro-L-arginine methyl ester (L-NAME), S-nitroso- $N$-acetyl-penicillamine (SNAP), $N$-acetyl-penicillamine (NAP), 8-bromoguanosine 3': 5'-cyclic monophosphate (8-br-cGMP), 2-phenyl-4,4,5,5-tetramethyl-imidazoline-1-oxyl 3-oxide (PTIO), and 1H-[1,2,4]oxadiazolo-[4,3-a]quinoxalin-1-one (ODQ). Chemicals were maintained in the dark and dissolved in crayfish saline to the required concentration immediately prior to application (within $5 \mathrm{~min}$ ), with the exception of ODQ, which was first dissolved in ethanol and then serially diluted in crayfish saline to a final concentration of ethanol of $<0.1 \%$. The recording chamber was constantly superfused with saline using a microtube pump (502S; Watson-Marlow), and the bathing solution changed at a rate of $10 \mathrm{ml} / \mathrm{min}$ for the application of drugs. The latency between the onset of drug application and 95\% applied concentration was approximately $80 \mathrm{~s}$ (Aonuma and Newland, 2001). Each preparation was exposed to drugs for $5 \mathrm{~min}$ and then washed with normal saline for $15 \mathrm{~min}$, and were tested only once with each compound, with the exception of control tests using D-arginine, D-NAME, and NAP, which were each followed by their active isomers. Chemical compounds were never reapplied to the same preparation. To rule out possible changes due to fatigue in the preparation additional sham controls were carried out by application of fresh saline for $5 \mathrm{~min}$, followed by a similar wash period of $15 \mathrm{~min}$ in normal saline.

\section{RESULTS}

\section{Local Reflex Responses of Nerve 3 Motor Neurons}

Electrical stimulation of sensory nerve 2 results in an increase in activity of some, but not all, motor neurons with axons in nerve 3 [Fig. 1(A)]. These motor neurons include five excitatory motor neurons that innervate the ventral rotator muscle, and over six that innervate the three promotor muscles of the exopodite (Larimer and Kennedy, 1969). The tonically active promotor motor neurons were inhibited during electrical stimulation [Fig. 1(A)]. The remaining large burst of activity therefore originates principally from the rotator motor neurons. These responses were still produced when the connectives between the sixth and fifth ganglia were severed and therefore constitute a simple local reflex response to the applied stimulus. To examine the change in this response over the time course of an experiment we used a sham control by replacing the saline in the recording chamber with fresh saline for $5 \mathrm{~min}$ and returning to the original saline for 15 min wash [Fig. 1(B)]. This change in saline had no statistically significant effect on the evoked spike frequency [ANOVA; $F_{1,3}=0.325 ; p$ $>0.05]$, but there was a slight, although not signifi- cant, run down in response frequency with time over the course of an entire experiment $(n=4$ animals $)$ [Fig. 1(C)].

\section{Effects of Endogenous NO on Local Reflexes}

Our previous experiments have suggested that a $\mathrm{NO} /$ cGMP signaling pathway is involved in the modulation of synaptic inputs to local circuits in the terminal abdominal ganglion (Aonuma and Newland, 2001). Using a number of pharmacological agents we have shown using a NO-specific indicator that we can experimentally manipulate NO levels in the terminal ganglion (Schuppe et al., 2002). We have used many of these same agents to analyze the effects of NO on the output properties of the local circuits, the reflex responses of nerve 3 motor neurons in response to nerve 2 stimulation. To increase the substrate available for endogenous NO synthesis we bath applied 5 $\mathrm{m} M \mathrm{~L}$-arginine for $5 \mathrm{~min}$. This produced a significant increase in the spike frequency of the evoked response from $106 \pm 6$ to $172 \pm 23 \mathrm{~Hz}$ [mean $\pm \mathrm{SEM}, n=6$ animals, ANOVA; $F_{1,6}=10.26 ; p<0.05$ ] [Fig. 2(A,B)]. Following 15 min wash in fresh saline the spike frequency of the evoked response returned to control levels $(101 \pm 22 \mathrm{~Hz})$ and was not significantly different from initial control levels [Fig. 2(B)]. As a control we applied the inactive isomer, D-arginine, at a similar concentration of $5 \mathrm{mM}(n=5$ animals $)$, which had no significant effect on the spike frequency of the evoked response [ANOVA; $F_{1,4}=1.455 ; p$ $>0.05$ ] [Fig. 2(C)].

Many of the motor neurons were spontaneously active prior to electrical stimulation (Fig. 1). Neither L-arginine, nor any of the other drugs tested in this study, had any consistent effect on the frequency of spontaneous spike activity.

Histochemical studies have shown that the enzyme responsible for NO synthesis, NOS, is present in a number of neurons in the terminal abdominal ganglion (Schuppe et al., 2001a). To decrease endogenous NO synthesis we applied the NOS inhibitor, L-NAME. Bath application of $5 \mathrm{~m} M$ L-NAME significantly decreased the spike frequency of the motor neurons from $230 \pm 46$ to $125 \pm 33 \mathrm{~Hz}[n=6$ animals, ANOVA; $F_{1,5}=10.352 ; p<0.05$ ] [Fig. $3(\mathrm{~A}, \mathrm{~B})]$. Following a further $15 \mathrm{~min}$ wash in fresh saline the response returned towards control levels (158 $\pm 40 \mathrm{~Hz}, n=6$ animals) [Fig. 3(B)]. Bath application of the inactive isomer, D-NAME, at a similar concentration of $5 \mathrm{~m} M$ had no significant effect [ANOVA; $F_{1,1}=0.325 ; p>0.05$ ] on the evoked responses ( $n=5$ animals) [Fig. 3(C)], al- 


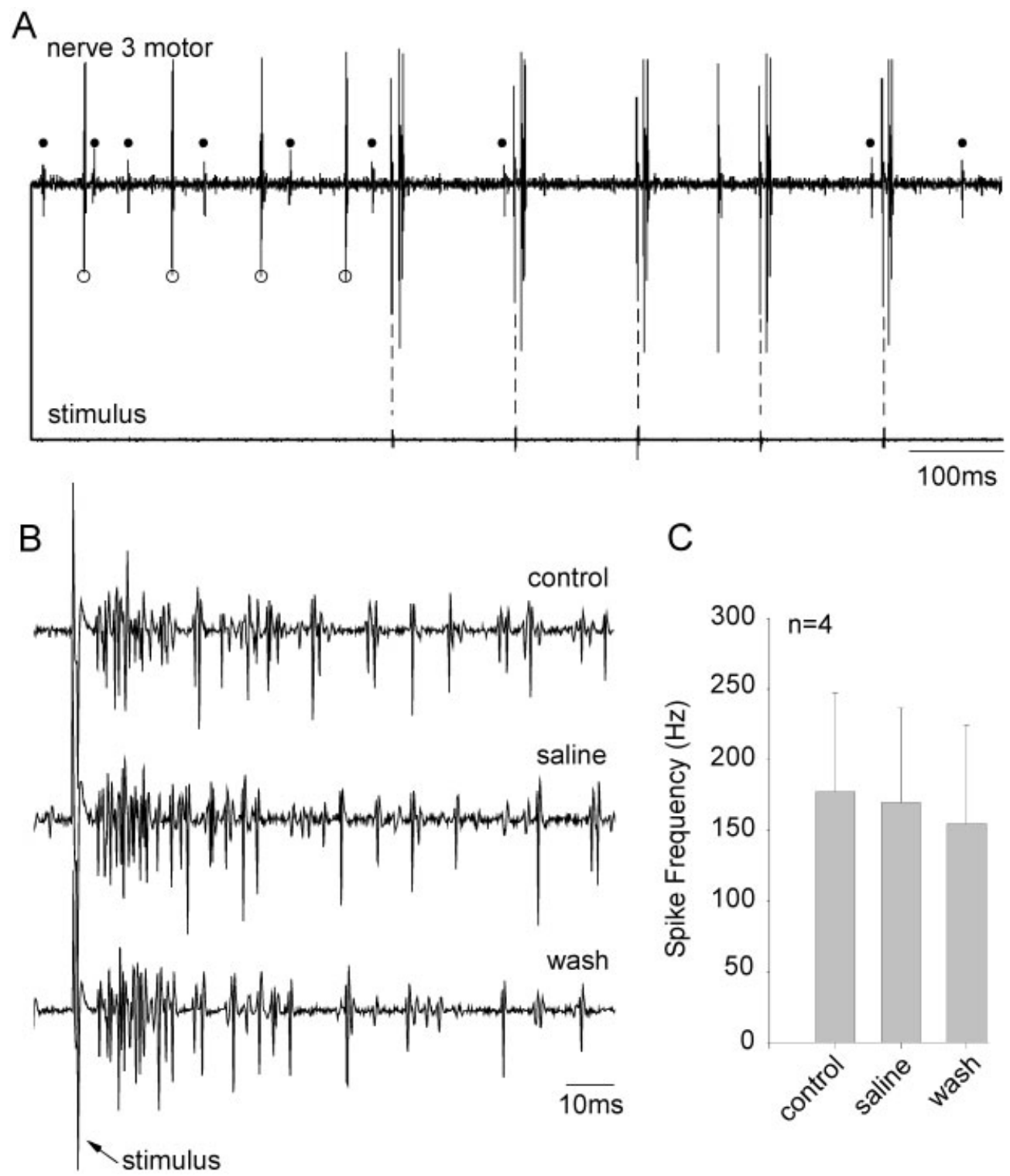

Figure 1 Local reflex responses of uropod motor neurons. (A) Electrical stimulation of the nerve 2 sensory bundle evokes a burst of activity of the rotator motor neurons recorded in the motor bundle of nerve 3. Five stimulus pulses were applied (bottom trace) and following each of the stimulus pulses was a burst of motor neuron activity. The spontaneous activity of two opener (promotor) motor neurons (indicated by the open and closed circles) was reduced in frequency. (B) Sham control responses showed that bathing the terminal ganglion in normal saline for 5 min (saline) caused no significant change in the frequency of the reflex response compared to control responses (control). Subsequent washing in fresh saline for a further $15 \mathrm{~min}$ (wash) also had no effect on the spike frequency. (C) Mean spike responses from four animals are shown before, after 5 min saline application, and after a further $15 \mathrm{~min}$ wash (data shown in this and subsequent figures are the means \pm SEM).

though the response showed some run down over time, as with controls (c.f. Fig. 1).

As an alternative method of reducing endogenous NO levels we bath applied $2.5 \mathrm{~m} M$ PTIO, a NO scavenger, for $5 \mathrm{~min}$, which significantly decreased the frequency of the evoked response from $168 \pm 36$ to $115 \pm 18 \mathrm{~Hz}\left[n=6\right.$ animals, ANOVA; $F_{1,5}$ $=6.919 ; p<0.05$ ] (Fig. 4). Responses failed to recover to control levels, although this may be due, in part, to the relatively short wash periods we used in 


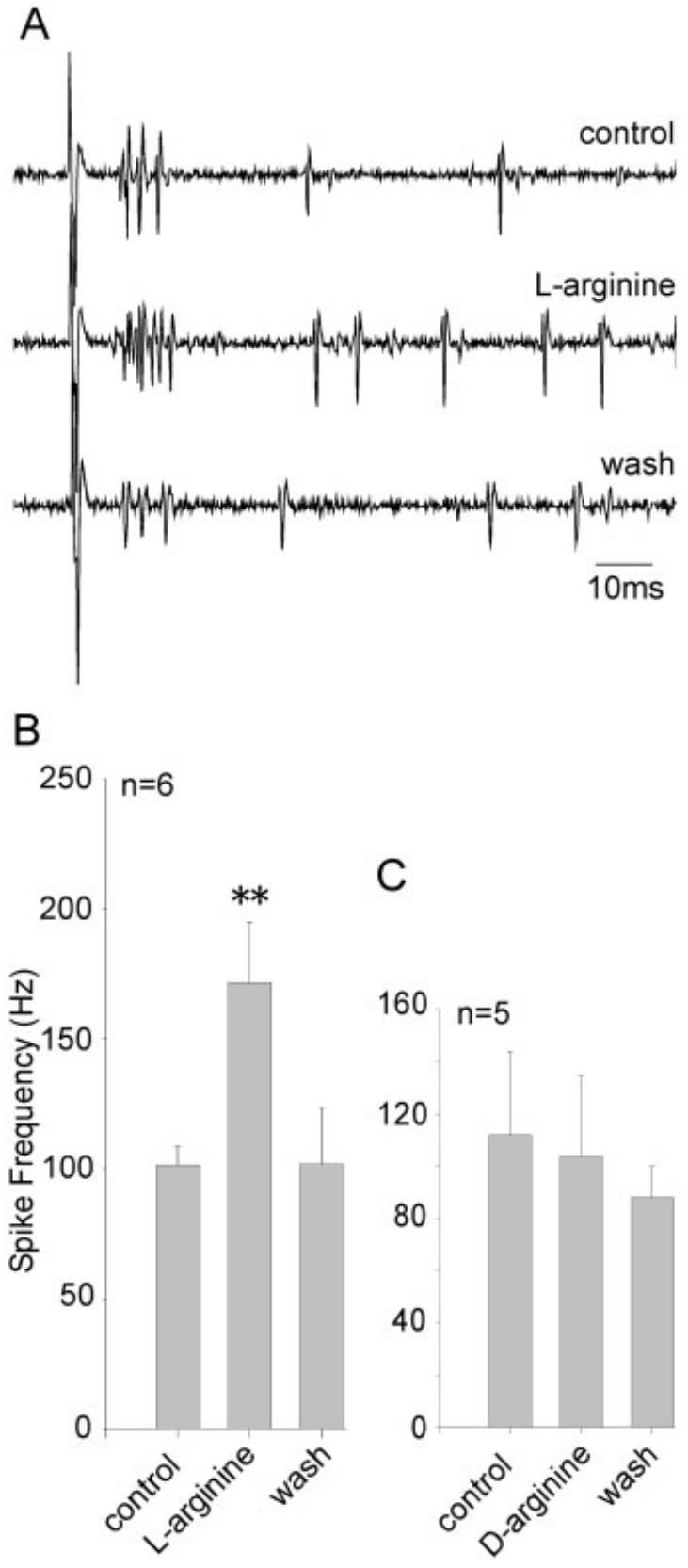

Figure 2 Effects of bath application of the substrate for NO synthesis, L-arginine, and its inactive isomer, Darginine, on the reflex responses of the uropod motor neurons. (A) An example showing that bath application of $5 \mathrm{mM}$ L-arginine caused a significant increase in the frequency of the evoked response after $5 \mathrm{~min}$ (Larginine), compared to control (control). The spike frequency of the response returned to control levels after a further 15 min wash in normal crayfish saline (wash). (B) Mean responses from six animals show that the application of L-arginine caused a statistically significant increase in spike frequency compared to control responses (**ANOVA, $p<0.05$ ). (C) Bath application of Darginine $(n=5)$ had no significant effect on the frequency of the evoked response (ANOVA, $p>0.05$ ).
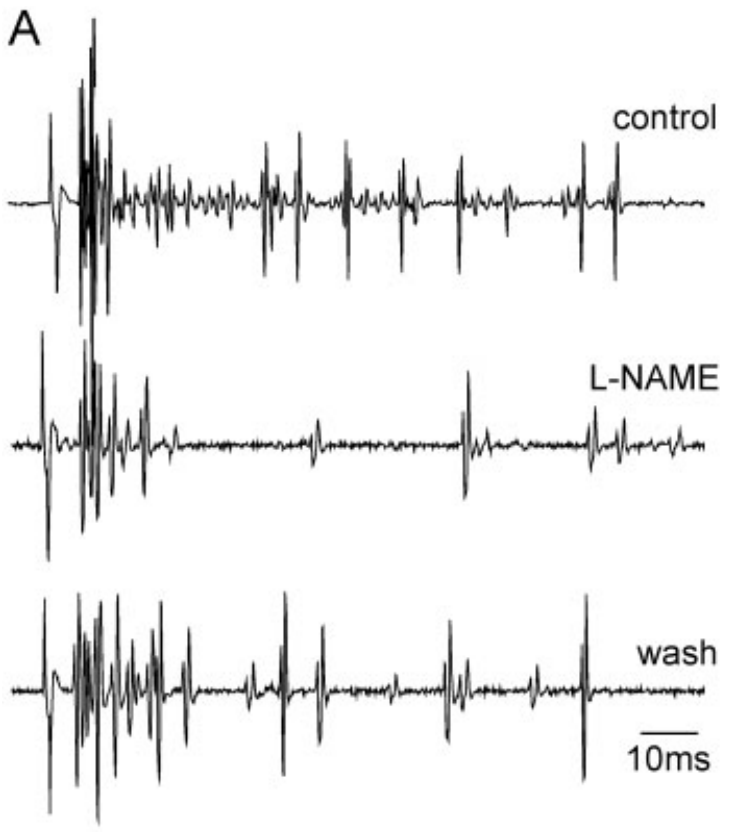

B

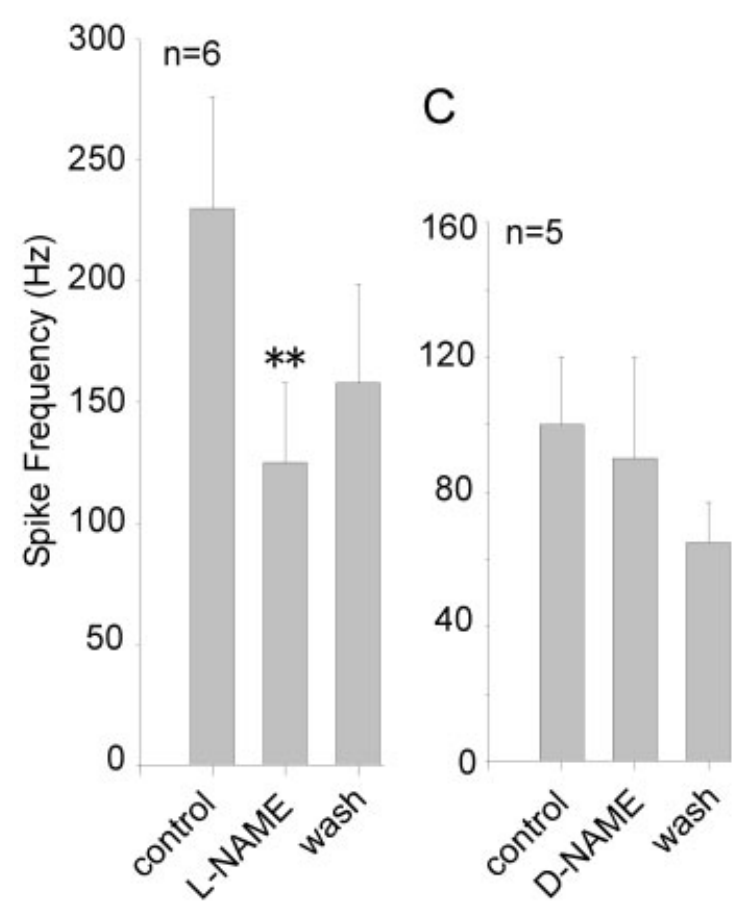

Figure 3 The NOS inhibitor, L-NAME, reduced the frequency of the evoked response. (A) An example showing that bath application of the $5 \mathrm{mM}$ L-NAME for $5 \mathrm{~min}$ significantly reduced the frequency of the evoked response. Following a further $15 \mathrm{~min}$ wash in normal saline the response returned to control levels. (B) Mean responses from six animals showing that L-NAME caused a significant reduction in spike frequency of approximately $50 \%$ (**ANOVA, $p<0.05$ ). (C) Bath application of $5 \mathrm{mM}$ D-NAME, an inactive isomer of L-NAME, had no significant effect on the evoked spike frequency $(n=5)$. 
A
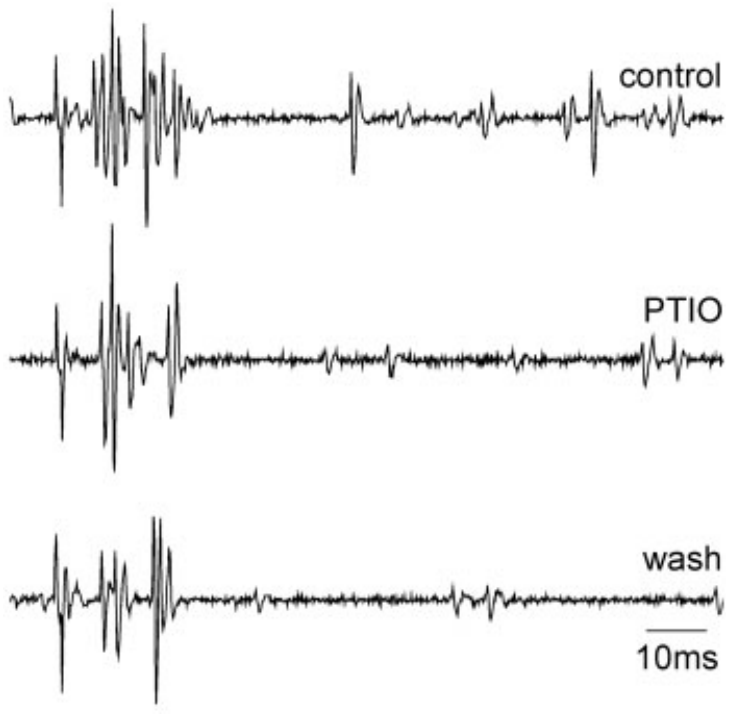

B

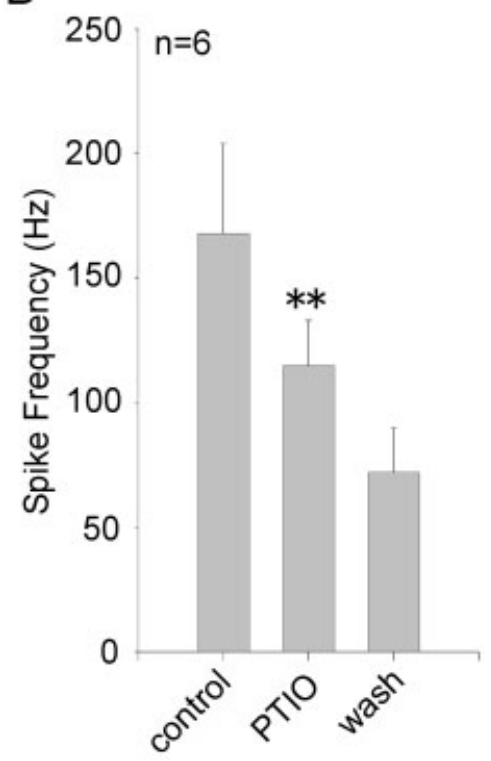

Figure 4 Effects of bath application of the NO scavenger PTIO on the reflex responses of the uropod motor neurons. (A) An example showing that bath application of $2.5 \mathrm{mM}$ PTIO caused a significant decrease in the frequency of the evoked response after 5 min (PTIO), compared to control (control), but showed little recovery following $15 \mathrm{~min}$ wash in normal crayfish saline (wash). (B) Mean responses from six animals show that the application of $2.5 \mathrm{mM}$ PTIO caused a statistically significant decrease in spike frequency compared to control responses (**ANOVA, $p<0.05$ ).

this study compared to our previous studies in which responses recovered to control levels after $30 \mathrm{~min}$ wash (Aonuma and Newland, 2001).

\section{Effects of Elevating Exogenous NO Levels}

Imaging studies using the NO donor, SNAP, have shown that it elevates NO levels in all cells in the terminal ganglion of the crayfish (Schuppe et al., 2002). We therefore analyzed the effects of SNAP on the local reflex responses of nerve 3 motor neurons during sensory stimulation (Fig. 5). Bath application of $400 \mu M$ SNAP for 5 min significantly increased the spike frequency of the evoked response from $63 \pm 13$ to $87 \pm 17 \mathrm{~Hz}\left[n=6\right.$ animals, ANOVA; $F_{1,5}$ $=22.273 ; p<0.05$ ] [Fig. 5(A,B)]. In contrast, bath application of $400 \mu M$ NAP for $5 \mathrm{~min}$, which is unable to release NO, had no significant effect on the evoked response ( $n=2$ animals) [Fig. 5(C)].

\section{Modulation of Response Is Mediated by NO-Induced cGMP Release}

NO can exert its effects through many pathways, including elevating cGMP levels through the action of the enzyme sGC. To determine whether NO in the terminal ganglion mediates its effects through sGC and cGMP release, we bath applied the specific inhibitor of sGC, ODQ. Bath application of $100 \mu M$ ODQ for $5 \mathrm{~min}$ significantly reduced the spike frequency of the motor response from $192 \pm 50$ to 92 $\pm 34 \mathrm{~Hz}\left[n=5\right.$ animals, ANOVA; $F_{1,4}=7.406 ; p$ $<0.05$ ] [Fig. 6(A,B)]. After a subsequent wash for 15 $\mathrm{min}$ in fresh saline the spike frequency of the response returned toward control levels $(117 \pm 33 \mathrm{~Hz}, n=5$ animals) [Fig. 6(B)].

To increase the levels of cGMP in the terminal ganglion we bath applied a membrane permeable analogue of cGMP, 8-br-cGMP, for $5 \mathrm{~min}$ at a concentration of $100 \mu M$. This bath application of 8-brcGMP significantly increased the spike frequency of the evoked response from $266 \pm 34$ to $314 \pm 30 \mathrm{~Hz}$ $\left[n=5\right.$ animals, ANOVA; $\left.F_{1,4}=9.071 ; p<0.05\right]$ [Fig. 6(C)]. During subsequent wash in fresh saline the spike frequency of the evoked response returned towards initial control levels [Fig. 6(C)].

To investigate further whether elevated NO levels act via sGC we bath applied $5 \mathrm{~m} M$ L-arginine simultaneously with $100 \mu M$ ODQ. After 5 min bath application we found no significant change in the spike frequency of the evoked responses ( $n=6$ animals) (Fig. 7). L-arginine $(5 \mathrm{~m} M)$ alone produced a 61 $\pm 18 \%$ increase in the spike frequency of the uropod motor neurons $(n=6)$, whereas $100 \mu M$ ODQ reduced the spike frequency by $46 \pm 11 \%(n=5)$. The simultaneous application of both compounds, however, produced a small reduction in spike frequency of only $2 \pm 2 \%(n=8)$ (Fig. 7$)$. 


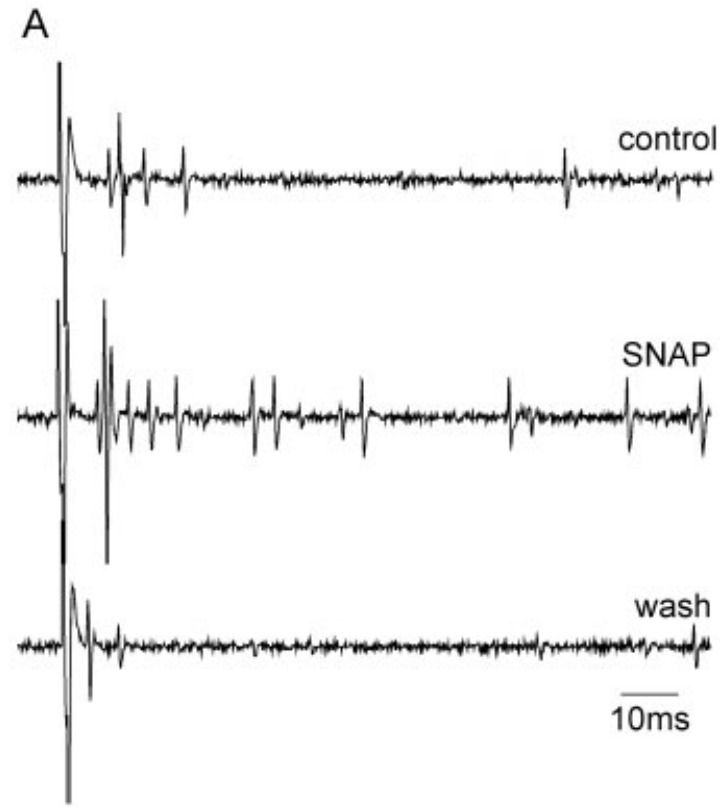

B

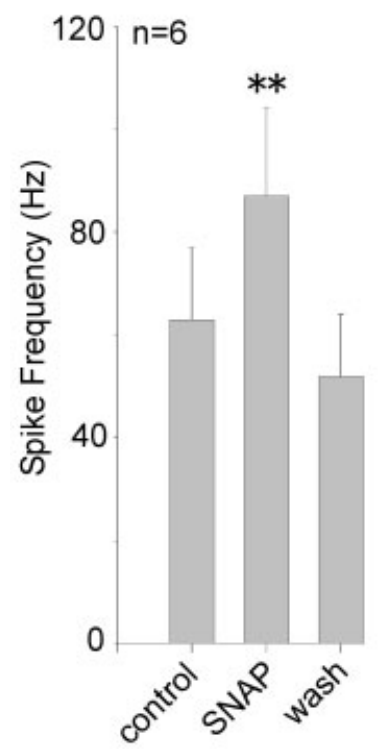

C

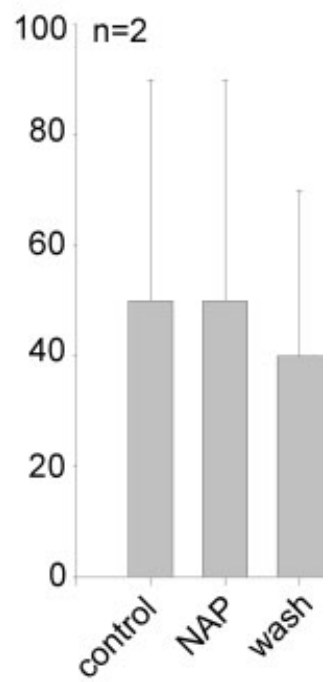

Figure 5 The NO donor SNAP increased the frequency of the evoked response. (A) An example showing that bath application of $400 \mu M$ SNAP for 5 min increased the spike frequency of the evoked response. After washing for $15 \mathrm{~min}$ in normal saline the response returned to control levels. (B) Mean responses from six animals showing that SNAP caused a significant increase in spike frequency of approximately 30\% (**ANOVA, $p<0.05$ ). (C) Bath application of $400 \mu M$ NAP had no significant effect on the evoked spike frequency $(n=2)$.

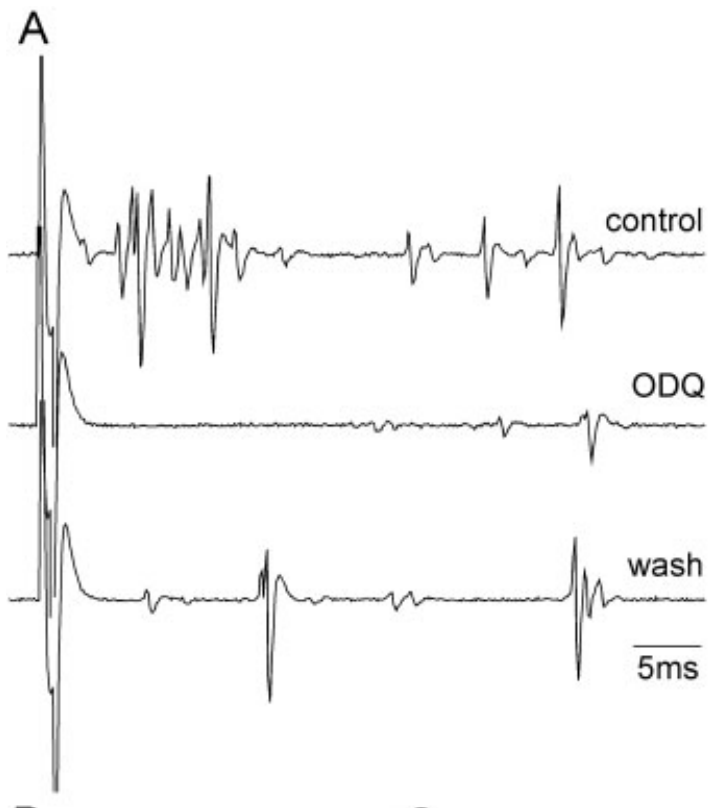

B
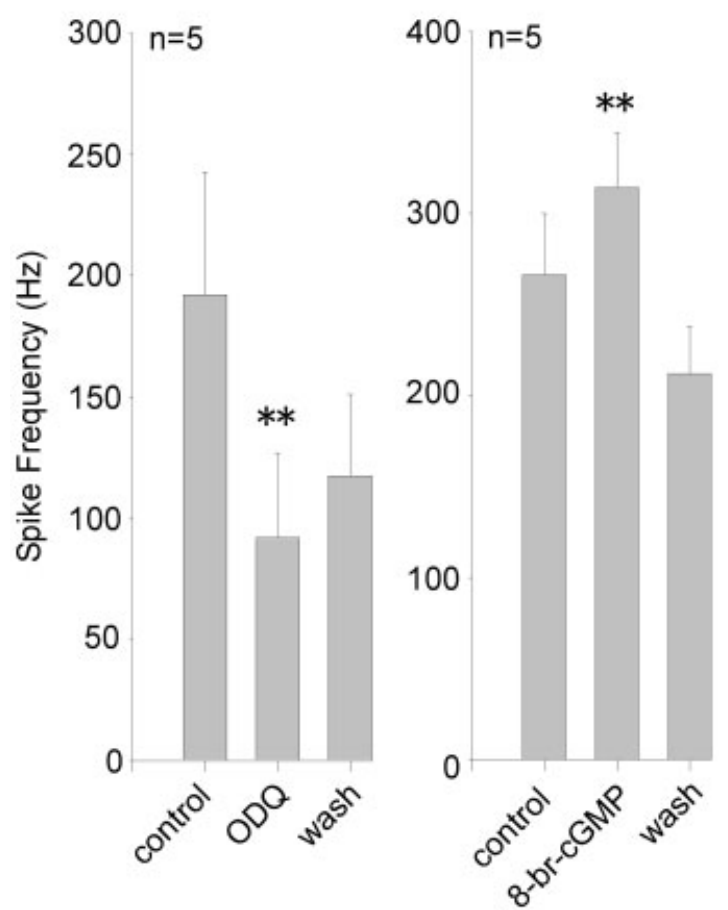

Figure 6 Effects of bath application of the sGC inhibitor ODQ and the cGMP analogue, 8-br-cGMP. (A) Bath application of 100 $\mu M$ ODQ almost completely abolished the spike response of the motor neurons after $5 \mathrm{~min}$, in this example. After a further $15 \mathrm{~min}$ wash in normal saline the response partly recovered. (B) Mean responses from five animals show that $100 \mu M$ ODQ caused a significant decrease in the spike frequency of response (**ANOVA, $p<0.05$ ) that partly returned to control levels after 15 min wash. (C) The cGMP analogue, $100 \mu M$ 8-br-cGMP, significantly increased the spike frequency of the response (**ANOVA, $p<0.05$ ), which recovered to control levels following 15 min wash in normal saline. 


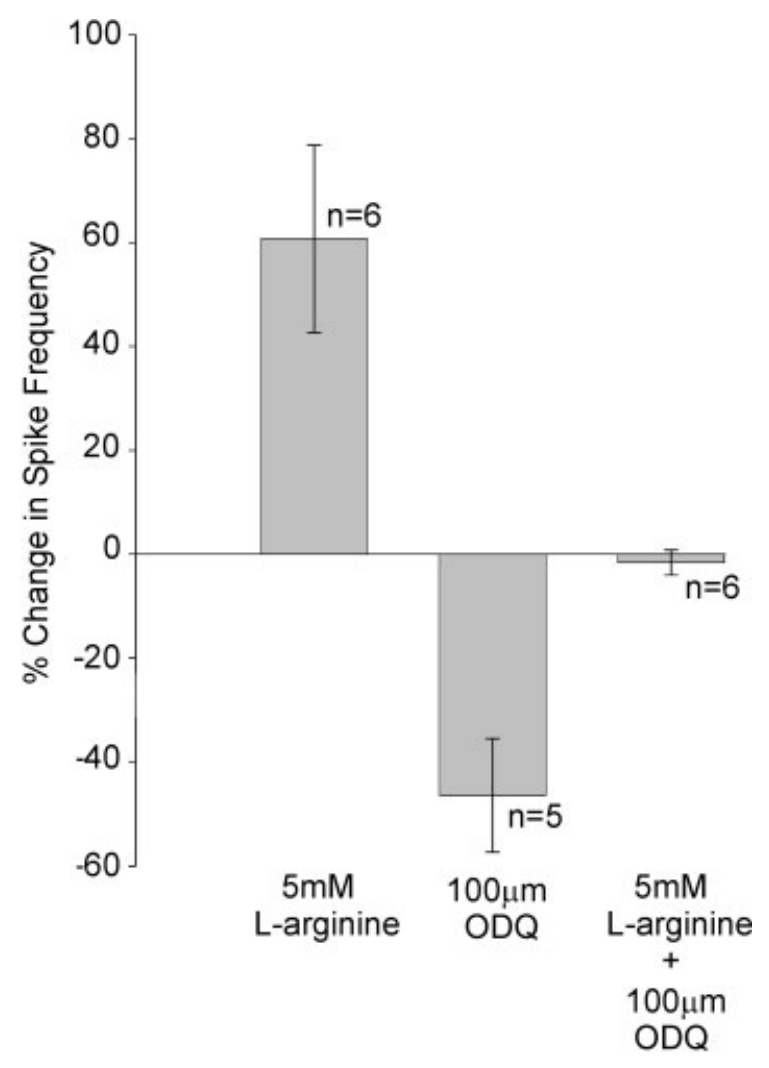

Figure 7 The L-arginine-induced increase in NO acts via sGC. Bath application of $5 \mathrm{mM}$ L-arginine alone for $5 \mathrm{~min}$ caused an increase in spike frequency of approximately $60 \%(n=6)$, while $5 \mathrm{~min}$ bath application of $100 \mu M$ ODQ alone decreased the frequency of the evoked response by approximately $46 \%(n=5)$. Simultaneous application of both drugs for $5 \mathrm{~min}$ had no significant effect on the frequency of the evoked response $(n=6)$.

\section{DISCUSSION}

\section{Local Reflex Responses of Uropod Motor Neurons}

The synaptic organization of the local circuits of crayfish closely resembles that of other invertebrates, in particular that of the locust, which has also been studied in detail (see Burrows, 1996). In crayfish, sensory hairs on the surface of the uropods, and internal joint receptors, are innervated by sensory neurons that arborize in the terminal abdominal ganglion, where they form monosynaptic connections with spiking local interneurons, intersegmental interneurons, and nonspiking interneurons (Nagayama, 2001; Newland et al., 2000). Likewise, in the locust exteroceptive and proprioceptive afferents form convergent monosynaptic connections with the same classes of interneurons (Burrows, 1987; Laurent and
Burrows, 1990; Siegler and Burrows, 1983). Stimulation of the hairs on the exopodite of crayfish evokes a reflexive closing of the appendage, brought about by an increase in the activity of the tonic closer motor neurons, and an inhibition of the tonic opener motor neurons (Nagayama et al., 1994). The reflex pattern of activity we observe in this study resembles that previously described, with a reduction in the spontaneous activity of promotor motor neurons, but a burst of activity in the rotator motor neurons. Our study clearly shows that the spike frequency of the reflex response of these motor neurons is regulated by endogenous levels of NO, as it is by the activity of other neuromodulators such as serotonin (Nagayama, 2002), presumably matching the pattern of motor activity to the precise conditions and requirements of the animal.

\section{Endogenous NO Levels Contribute to Reflex Responses}

The results we have obtained in this study allow us to compare the effects of pharmacological agents on the responses of uropod motor neurons with the resulting changes in NO levels, previously established through imaging using the NO-specific indicator 4,5-diaminofluoroscein (Nakatsubo et al., 1998; Schuppe et al., 2002). Our pharmacological results are consistent with modulation of the local reflexes occurring through a NO/cGMP signaling pathway. Bath application of drugs that regulate the levels of NO in the terminal ganglion also regulate the strength of local reflex responses of the uropod motor neurons. For example, up-regulating endogenous or exogenous NO levels by applying L-arginine or SNAP leads to an enhancement of the reflex response in rotator motor neurons, whereas decreasing NO levels with the NOS inhibitor, L-NAME, or the NO scavenger, PTIO, reduces the reflex responses (Fig. 8). Moreover, bath application of the sGC inhibitor, ODQ, and an analogue of cGMP indicate that one of the targets of NO in the terminal ganglion is the enzyme sGC, and that the actions of NO are mediated through cGMP (Fig. 8). Such a suggestion has been alluded to in our previous studies, although we had no direct evidence that the increased levels of $\mathrm{NO}$ were acting via sGC (Aonuma and Newland, 2001, 2002). By applying L-arginine and ODQ simultaneously to the terminal ganglion, the L-arginine-induced increase in reflex response was blocked by ODQ, implying that NO exerts its modulatory effects through sGC and cGMP, which provides the important evidence that has been lacking until now. 


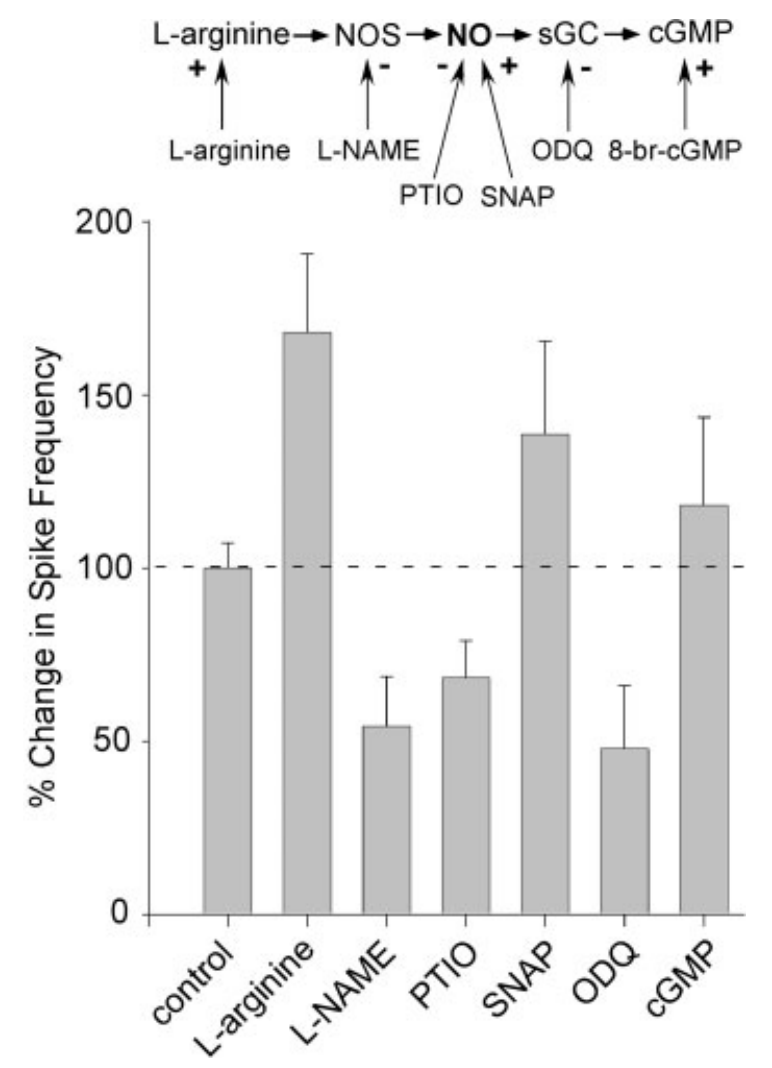

Figure 8 Summary of the effects of NO on the spike frequency of the uropod motor neurons. The means \pm SEM of the percentage changes in spike frequency are shown for each of the drugs that elevate or decrease either $\mathrm{NO}$ or cGMP, taken from the data in previous figures. Pearson chi-square analysis shows that there is an association between drug application and reflex responses of the uropod motor neurons $\left(\chi^{2}=34\right.$, d.f. $\left.=5, p<0.001\right)$. The inset shows the NO/cGMP signaling pathway. Plus and minus signs refer to the increases and decreases, respectively, of the effects of the drugs on the reflex response.

\section{NO Action in Local Circuits}

How might NO exert its effects on the local circuits? In the locust, detailed studies by Ott and Burrows (1998) and Ott et al. (2000) have shown that specific local and intersegmental interneurons are potential sources of NO. Close parallels exist in the local circuits of crayfish in which similar classes of neurons are strongly labeled using NO-specific indicators (Schuppe et al., 2002). Moreover, double labeling studies combining NADPHd histochemistry and intracellular dye injection have shown that many of the 65 pairs of ascending interneurons in the terminal abdominal ganglion synthesize NO (Schuppe et al., 2001b). Recent pharmacological studies have shown that sensory inputs to the ascending interneurons, activated by means of electrical stimulation, are sub- ject to modulation by NO. Ascending interneurons whose inputs are enhanced by NO have been defined as class I interneurons, whereas ascending interneurons whose inputs are depressed by NO have been defined as class II interneurons (Aonuma and Newland, 2001). Given the apparent similarities between the local circuits of insects (Burrows, 1996) and crustaceans (Nagayama, 2002), such a similar organization is not surprising; however, the similarities end when the potential targets of NO are considered. Ott et al. (2001) used two complimentary approaches to determine the targets for NO in the locust thoracic nervous system: immunolocalization of the $\alpha$-subunit of sGC and of NO-induced cGMP. Their studies indicate that sensory neurons and motor neurons are the most likely targets of NO in the thoracic ganglia, a result supported by Rast (2001), who showed that NO-induced cGMP immunoreactivity was also present in sensory and motor neurons in the suboesophageal ganglion. Moreover, cGMP has been implicated in the early modulation of sensory signals in many different systems (Bacigalupo et al., 1995; Bicker and Schmachtenberg, 1997; Ott et al., 2000). In crayfish, physiological studies have also pointed to a presynaptic site of action of NO on mechanosensory neurons, because NO had no effect on the membrane potential or input impedances of ascending interneurons (Aonuma and Newland, 2001) or on spiking local interneurons (Aonuma and Newland, 2002). Given the apparent similarity with the synaptic organization of the local circuits of insects, it is somewhat surprising that a recent study by Aonuma (2002) described a strikingly different pattern of NO-induced cGMP staining in the terminal abdominal ganglion of crayfish, in which specific local and intersegmental interneurons are described as being potential targets for NO. This suggests that a proposed model for NO action, in which activity-dependent NO acts on the terminals of neighboring sensory neurons (Ott et al., 2000,2001 ), is unlikely in the crayfish terminal ganglion. The fundamental basis of the proposed model resided in the precise topographic mapping of the mechanosensory neurons (Newland, 1991; Newland et al., 2000), so that the localized release of NO could modulate the strength of synaptic interactions in the near vicinity. Such maps also exist in the terminal ganglion of the crayfish (Kondoh and Hisada, 1986; Nagayama and Newland, 1993), and it had been thought that a similar model could also be likely in crayfish (Aonuma and Newland, 2001). It is possible that intrinsic local circuit neurons are the targets for NO, however, it is difficult to resolve how they would be likely to mediate the up- and down-regulation of synaptic inputs to ascending interneurons (Aonuma 
and Newland, 2001), the depression of spiking local interneurons, and the down-regulation of presynaptic inhibition (Schuppe and Newland, 2004), all by virtue of the patterns of connections they make with these classes of interneurons. It is worth noting, however, that the cGMP staining pattern observed by Aonuma (2002) is not the exception to the rule, because Bicker et al. (1996) showed that in the olfactory system of locusts NO also acts on intrinsic interneurons. Clearly there is much still to understand as to where NO is mediating these effects within the terminal ganglion and the precise mechanisms of its action, or indeed where it is acting. One obvious next step would be to use the approach taken by Ott et al. (2000) and to analyze the distribution of the $\alpha$-subunit of sGC and compare it with the NO-induced cGMP staining patterns.

\section{REFERENCES}

Aonuma H. 2002. Distribution of NO-induced cGMP-like immunoreactive neurons in the abdominal nervous system of the crayfish, Procambarus clarkii. Zool Sci 19: 969-979.

Aonuma H, Newland PL. 2001. Opposing actions of nitric oxide on synaptic inputs of identified interneurones in the central nervous system of the crayfish. J Exp Biol 204: 1319-1332.

Aonuma H, Newland PL. 2002. Synaptic inputs onto spiking local interneurons in crayfish are depressed by nitric oxide. J Neurobiol 52:144-155.

Bacigalupo J, Bautista DM, Brink DL, Hetzer JF, O'Day PM. 1995. Cyclic-GMP enhances light-induced excitation and induces membrane currents in Drosophila retinal photoreceptors. J Neurosci 15:5040-5050.

Bicker G, Schmachtenberg O. 1997. Cytochemical evidence for nitric oxide/cyclic GMP signal transmission in the visual system of the locust. Eur J Neurosci 9:189-193.

Bicker G, Schmachtenberg O, de Vente J. 1996. The nitric oxide/cyclic GMP messenger system in olfactory pathways of the locust brain. Eur J Neurosci 8:2635-2643.

Bredt DS, Snyder SH. 1989. Nitric oxide mediates glutamate-linked enhancement of cGMP levels in the cerebellum. Proc Natl Acad Sci USA 86:9030-9033.

Breer H, Shepherd GM. 1993. Implications of the NO/ cGMP system for olfaction. Trends Neurosci 16:5-9.

Burrows M. 1987. Parallel processing of proprioceptive signals by spiking local interneurons and motor neurons in the locust. J Neurosci 7:1064-1080.

Burrows M. 1996. The Neurobiology of an Insect Brain. Oxford: Oxford University Press. 682 p.

Cudeiro J, Rivadulla C. 1999. Sight and insight-on the physiological role of nitric oxide in the visual system. Trends Neurosci 22:109-116.

Elphick MR, Williams L, O'Shea M. 1996. New features of the locust optic lobe: Evidence of a role for nitric oxide in insect vision. J Exp Biol 199:2395-2407.

Gelperin A, Kao JPY, Cooke IRC. 2001. Gaseous oxides and olfactory computation. Am Zool 41:332-345.

Kemenes I, Kemenes G, Andrew RJ, Benjamin PR, Michael O'Shea, M. 2002. Critical time-window for NO-cGMPdependent long-term memory formation after one-trial appetitive conditioning. J Neurosci 22:1414-1425.

Kendrick KM, Guevara-Guzman R, Zorrila J, Hinto MR, Broad KD, Mimmack M, Ohkura S. 1997. Formation of olfactory memories mediated by nitric oxide. Nature 366 : $670-674$.

Kiedrowski L, Costa E, Wrobleski JT. 1992. Glutamate receptor agonists stimulate nitric-oxide synthase in primary cultures of cerebellar granule cells. Mol Pharmacol 41:779-784.

Kondoh Y, Hisada M. 1986. Neuroanatomy of the terminal (sixth abdominal) ganglion of the crayfish, Procambus clarkii. Cell Tissue Res 243:273-288.

Larimer JL, Kennedy D. 1969. Innervation patterns of fast and low muscle in the uropods of crayfish. J Exp Biol 51:119-133.

Laurent G, Burrows M. 1990. A population of ascending intersegmental interneurones in the locust with mechanosensory inputs from a hind leg. J Comp Neurol 275: $1-12$.

McLean DL, Sillar KT. 2000. The distribution of NADPHdiaphorase-labelled interneurons and the role of nitric oxide in the swimming system of Xenopus laevis larvae. J Exp Biol 203:705-713.

Moncada S, Palmer RMJ, Higgs EA. 1991. Nitric oxide: physiology, pathophysiology, and pharmacology. Pharmacol Rev 43:109-142.

Müller U. 1996. Inhibition of nitric oxide synthase impairs a distinct form of long-term memory in the honeybee Apis mellifera. Neuron 16:541-549.

Nagayama T. 1997. Organization of exteroceptive inputs onto nonspiking local interneurons in the crayfish terminal abdominal ganglion. J Exp Zool 283:541-547.

Nagayama T. 2001. Synaptic organization of local circuit neurons in the terminal abdominal ganglion of crayfish. In: Wiese K, editor. The Crustacean Nervous System. Berlin: Springer Verlag, p 591-600.

Nagayama T. 2002. Serotonergic modulation of non-spiking local interneurons in the terminal abdominal ganglion of the crayfish. J Exp Biol 205:3067-3076.

Nagayama T, Namba H, Aonuma H. 1994. Morphological and physiological bases of crayfish local circuit neurones. Histol Histopath 9:791-805.

Nagayama T, Newland PL. 1993. A sensory map based on velocity threshold of sensory neurones from a chordotonal organ in the tailfan of the crayfish. J Comp Physiol 172:7-15.

Nakatsubo N, Kojima H, Kikuchi K, Nagoshi H, Hirata Y, Maeda D, Imai Y, Irimura T, Nagano T. 1998. Direct evidence of nitric oxide production from bovine aortic endothelial cells using new fluorescence indicators: diaminofluoresceins. FEBS Lett 427:263-266. 
Newland PL. 1991. Morphology and somatotopic organisation of the central projections of afferents from tactile hairs on the hind leg of the locust. J Comp Neurol 312:493-508.

Newland PL. 2001. The role of proprioception in the tailfan of the crayfish. In: Wiese K, editor. The Crustacean Nervous System. Berlin: Springer Verlag, p 601-609.

Newland PL, Rogers SM, Gaaboub I, Matheson T. 2000. Parallel somatotopic maps of gustatory and mechanosensory neurons in the central nervous system of an insect. J Comp Neurol 425:82-96.

Ott SR, Burrows M. 1998. Nitric oxide synthase in the thoracic ganglia of the locust: Distribution in the neuropiles and morphology of neurones. J Comp Neurol 395: 217-230.

Ott SR, Burrows M, Elphick MR. 2001. The neuroanatomy of nitric oxide-cyclic GMP signaling in the locust: functional implications for sensory systems. Amer Zool 41: 321-331.

Ott SR, Jones IW, Burrows M, Elphick MR. 2000. Sensory afferents and motor neurons as targets for nitric oxide in the locust. J Comp Neurol 422:521-532.

Philippides A, Husbands P, O'Shea M. 2000. Four-dimensional neuronal signaling by nitric oxide: a computational analysis. J Neurosci 20:1199-1207.

Rast GF. 2001. Nitric oxide induces centrally generated motor patterns in the locust suboesophageal ganglion. $\mathbf{J}$ Exp Biol 204:3789-3801.

Schmachtenberg O, Bicker G. 1999. Nitric oxide and cyclic GMP modulate photoreceptor cell responses in the visual system of the locust. J Exp Biol 202:13-20.
Schmidt HHH, Walter U. 1994. NO at work. Cell 78:919925.

Scholtz NL, de Vente J, Truman JW, Graubard K. 2001. Neural network partitioning by NO and cGMP. J Neurosci 21:1610-1618.

Schuppe H, Aonuma H, Newland PL. 2001a. NADPHdiaphorase histochemistry in the terminal abdominal ganglion of the crayfish. Cell Tissue Res 303:289-299.

Schuppe H, Aonuma H, Newland PL. 2001b. Distribution of NADPH-diaphorase positive ascending interneurons in the crayfish terminal abdominal ganglion. Cell Tissue Res 305:135-146.

Schuppe H, Araki M, Aonuma H, Nagayama T, Newland PL. 2004. Effects of nitric oxide on proprioceptive signaling. Zool Sci 21:1-5.

Schuppe H, Cuttle M, Chad JE, Newland PL. 2002. 4,5diaminofluoroscein imaging of nitric oxide synthesis in crayfish terminal ganglia. J Neurobiol 53:361-369.

Schuppe H, Newland PL. 2004. Nitric oxide modulates presynaptic afferent depolarization of mechanosensory neurons. J Neurobiol, to appear.

Siegler MVS, Burrows M. 1983. Spiking local interneurons as primary integrators of mechanosensory information in the locust. J Neurophysiol 50:1281-1295.

Stuehr DJ, Griffith OW. 1992. Mammalian nitric oxide synthases. Adv Enzymol 65:287-346.

van Harreveld A. 1936. A physiological solution for freshwater crustaceans. Proc Soc Exp Biol 34:428-432.

Wink DA, Hanbauer I, Krishna MC, DeGraff W, Gamson J, Mitchell JB. 1993. Nitric-oxide protects against cellular damage and cytotoxicity from reactive oxygen species. Proc Natl Acad Sci USA 90:9813-9817. 